\title{
TUNABLE CAPACITORS WITH PROGRAMMABLE CAPACITANCE-VOLTAGE CHARACTERISTIC
}

\author{
Elmer S. Hung and Stephen D. Senturia \\ Massachusetts Institute of Technology \\ Cambridge, MA 02139
}

\begin{abstract}
We report the design and fabrication of a micromechanical tunable capacitance device with a lithographically-programmable capacitance-voltage (CV) characteristic. Device prototypes utilizing a contact "zipping" actuator achieve a $25 \%$ tuning range with a linear C-V characteristic. Three significant results are described: (1) realization of a device with a large capacitive tuning range and programmable $\mathrm{C}-\mathrm{V}$ device characteristics, (2) development of a stable zipping actuator using flat structures, and (3) development of an automated meshed simulation/optimization technique used to design the device.
\end{abstract}

\section{INTRODUCTION}

Recent growth in the wireless communications industry has spawned tremendous interest in the development of highly-integrated transceiver solutions. Microelectromechanical (MEMS) tuning elements have received increasing attention for integrating off-chip components in order to reduce the fabrication cost, size, and complexity and increase the power efficiency of wireless devices.

For example, voltage-controlled oscillators (VCOs) traditionally require an off-chip LC tank using a tunable capacitor (a varactor) for frequency tuning. Fabricating the LC tank on chip requires the development of lowloss monolithic inductors and varactors in order to meet phase noise requirements. VCOs have been constructed by Young and Boser $[1,2]$ using micromechanical varactors consisting of an electrostatically-actuated aluminum plate suspended over a fixed bottom plate fabricated in a CMOS-compatible process. The two plates are not allowed to contact each other, so the tuning range is limited to the top $1 / 3$ of the gap, due to the electrostatic pull-in instability.

Other variable capacitance MEMS devices for RF systems include RF switches [3-5] for use in phase shifters and steerable antenna arrays. Although these switches are not designed for analog capacitance tuning, large on/off capacitance changes can be obtained by electrostatically pulling down one conductive diaphragm electrode onto a thin insulating layer deposited on top of the second electrode of a two-plate capacitor [5].

In this paper, we report the design and fabrication of analog-tuned micromechanical varactors based on a contact electrostatic "zipper" actuator. Zipper actuators are attractive for varactor applications for several reasons.

First, like MEMS RF switches, a large ratio of available gap sizes is used to obtain increased capacitive tuning ranges and large capacitances per unit area. However, unlike previous RF switches or analog-tuned varactors, the zipper varactors are designed to always operate in a contact "zipping" mode, providing analog capacitance tuning without significant limitations from electrostatic pull-in instabilities.

In addition, the specific capacitance-voltage (C-V) characteristic of the device can be tailored by lithographically programming the geometry of an electrode. The specific geometry that is necessary to achieve a desired $\mathrm{C}-\mathrm{V}$ characteristic is determined by an optimization requiring meshed simulations of a coupled electromechanical problem involving contact mechanics.

\section{DEVICE DESCRIPTION}

The zipping actuator varactor (Fig. 1) consists of a conductive cantilever beam over a shaped bottom electrode. The capacitance between the beam and the bottom electrode is controlled by an applied DC voltage, and the $\mathrm{C}-\mathrm{V}$ characteristic of the device is determined by the geometry of the bottom electrode.

When an increasing voltage is applied between the beam and the bottom electrode, the beam first bends downward, then collapses toward the substrate due to the electrostatic pull-in effect. Dimples in the cantilever hold the beam a small distance off the bottom electrode. At first, only the beam tip contacts the substrate, but as additional voltage is applied, the tip flattens and the beam "zips" along the substrate toward the cantilever support, increasing the area of the beam close to the bottom electrode, thus changing the device capacitance (see Fig. 2).

The device is designed to operate in the "zipping" regime (Fig. 2(c)) with the actuator in contact with the substrate. Electrostatic zipper actuators are attractive for a variety of applications because they provide large, stable displacements. Actuation force can be comparatively small due to small gaps and the zipping action of electrodes that are curved during operation. Previous efforts to fabricate zippers relied on the fabrication of curved electrodes [6-8]. Gilbert and Senturia [9] proposed a zipper actuator for use in torsional mirrors involving flat electrodes separated by a dielectric; however, no devices were fabricated.

As in previous work on zippers [6-9], the first versions of the varactors were fabricated using a dielectric to isolate the two electrodes. However, hysteresis was observed in these devices, which we attribute to trapped charge on the surfaces of the dielectric. In order to eliminate this effect, the dielectric between the electrodes was removed and replaced by an air gap defined by dimple spacers. The dimples also help reduce any other adhesion effects due to surface contact. In addition, the cantilever and substrate are DC grounds, minimizing the potential difference across the dielectric between the beam and the substrate. The device is actuated by applying a DC bias to the bottom electrode.

Prototype devices were fabricated using the MUMPs process from MCNC [10]. With the MUMPs process, 


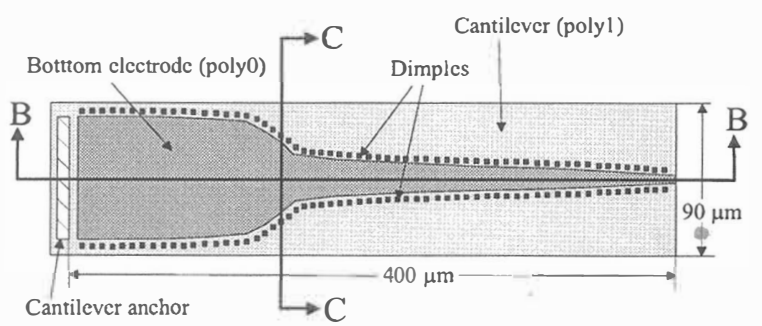

(a) Top View

Bottom electrode (poly 0)

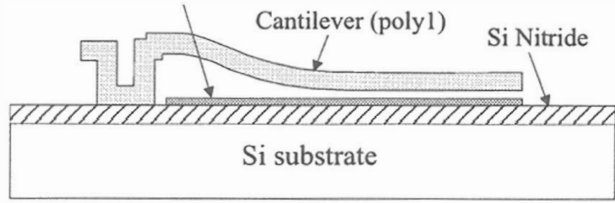

(b) Cross-section B (under actuation)

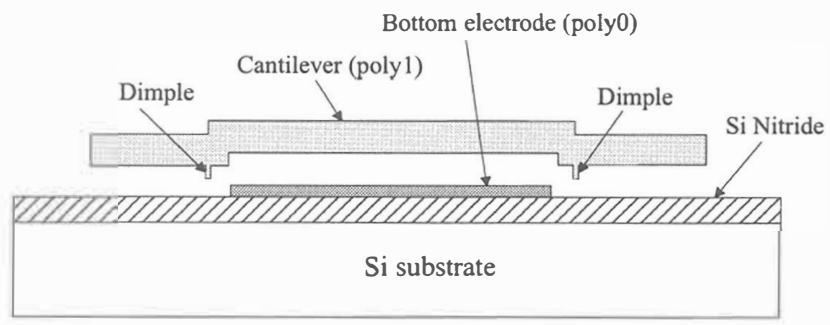

(c) Cross-section $\mathrm{C}$

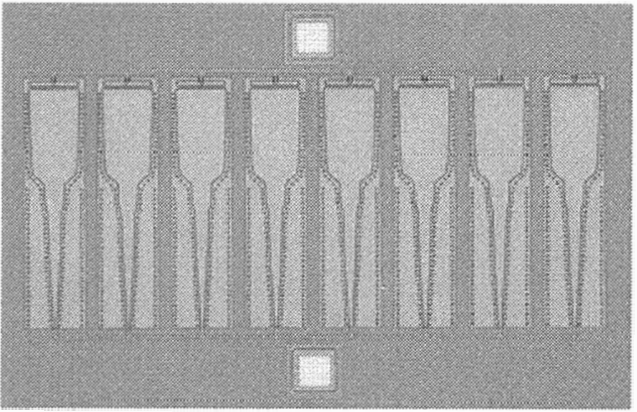

(d)

Figure 1. Tunable capacitance device. (a) Top view showing a shaped bottom electrode underneath a rectangular cantilever beam. (b) Lengthwise cross-section of beam under actuation in the zipping regime. The air gap under the end of the beam is defined by dimple spacers. (c) Width cross-section showing the dimple spacers. (d) Photograph of device.

the cantilever and bottom electrode are both polysilicon. The nominal beam thickness and gap size are $2 \mu \mathrm{m}$ and the dimple depth is $0.75 \mu \mathrm{m}$. The MUMPs process is not optimal for varactor fabrication. The default thickness values and dimple depth severely limits the design space. Also, metalized structures will ultimately be necessary to minimize resistive losses for many RF applications. However, MUMPs was chosen for prototyping convenience in order to test the basic actuation principle and verify the capacitance-voltage characteristics of the varactors.

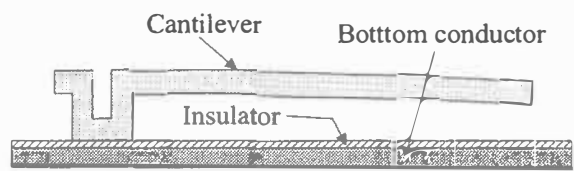

(a) Cantilever bending (before pull-in)

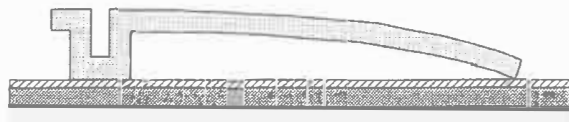

(b) Cantilever tip pull-in

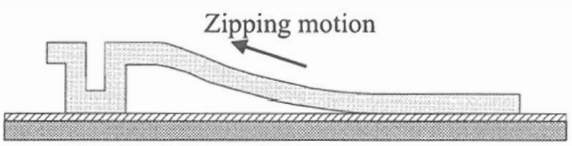

(c) Zipping regime

Figure 2. Zipper actuator operation with increasing voltage applied between the cantilever and bottom conductor. (a) At first, the beam bends stably. (b) The beam suddenly crashes to the substrate, supported by the beam tip. (c) The tip flattens and the beam "zips" along in contact with the substrate. In the varactors, the insulating layer is replaced by an air gap defined by dimple spacers in order to reduce hysteretic effects of dielectric charging and stiction.

\section{SIMULATION}

Because of the computational demands of optimization, it is important to be able to simulate the device quickly as well as accurately. The deflection of the cantilever is modeled by an elastic beam equation with electrostatic force and a contact force when the beam touches the substrate:

$$
E I \frac{\partial^{4} z}{\partial x^{4}}=F_{\text {elec }}+F_{\text {bot }}
$$

where $z(x)$ is the height of the lower beam above the bottom electrode, $x$ is the distance along the length of the beam, $E$ is the elastic modulus of polysilicon, $I=$ $w h^{3} / 12$ is the moment of inertia of the beam, $w$ is the width of the beam, $h$ is the thickness of the beam, $F_{b o t}$ is the force exerted on the beam by the substrate once the beam touches down, and $F_{\text {elec }}$ is the electrostatic force per unit length. If fringing fields are neglected we can approximate:

$$
F_{\text {elec }}=-\frac{\epsilon_{0} w(x) V^{2}}{2 z^{2}}
$$

where $w(x)$ represents the shape of the bottom electrode and $V$ is the applied voltage. The cantilever support is assumed to have perfectly clamped boundary conditions. Eq. (1) is simulated using finite differences. Full $3 D$ simulations [11] would produce greater accuracy, but the computation time would be prohibitive, since hundreds or thousands of beam deflection profiles must be computed for each design.

The contact condition $\left(F_{b o t}\right)$ adds significant complexity to the simulation. It requires solving both for the contact area and force simultaneously with the beam equation. This is accomplished by using a time-based relaxation technique. A hard contact condition is assumed 
constraining $z(x) \geq d$ for all $x$ where $d$ is the dimple depth. No sliding is allowed. Note that an air gap remains between the beam and bottom electrode when the dimples are in contact, so there is some unmodeled deflection of the beam along the width of the beam. Varactors must be designed so that the deflection along the width does not cause the beam to contact the bottom electrode.

The $\mathrm{C}-\mathrm{V}$ characteristic of the device is computed by simulating the deflection profile of the beam at several different voltages and calculating the capacitance at each voltage according to the parallel-plate formula (i.e., neglecting fringing):

$$
C(V)=\int \frac{\epsilon_{0} w(x)}{z(x, V)} d x .
$$

\section{OPTIMIZATION}

Previous efforts to automate the design of micromechanical devices have focused on the optimization of a few parameters of lumped-element models [12]. Electrode shaping and optimization techniques for comb drives have also recently been reported [13].

The design of the varactor requires meshed simulation and optimization of the continuous shape of an electrode. For the varactor, it would be quite difficult to design the device without the use of a significant simulation and optimization effort, since the resulting electrode shapes are not trivial.

The inputs to the optimization routine consist of geometric specifications including the length $L$ and width $W$ of the cantilever device as well as the optimization merit function to use. The merit function $M: E \rightarrow \mathrm{R}$ takes a bottom electrode geometry representation, $e \in E$, and returns a scalar evaluation of how good the design is, where $E$ is the space representing possible bottom electrode widths $w(x)$. In defining the merit function, there is a choice in how to represent electrode geometry. For example, $w(x)$ could be represented by a parameterized family of functions [13], or a discretized approximation. In addition, one can also choose the range of operating voltages that should be targeted for device operation.

For example, for the first prototype varactors, we attempt to design devices with a linear $\mathrm{C}-\mathrm{V}$ characteristic while maximizing the capacitive tuning range. Electrode geometry $e \in E=\mathrm{R}^{\mathrm{N}}$ is represented by a simple $N$-point discretization of the width function $w(x)$. A specific voltage range is input as the target voltage range for linear $\mathrm{C}-\mathrm{V}$ behavior. This voltage range is chosen so that it is possible for the device to reach the zipping regime with the given geometrical constraints.

The merit function for this design is computed as follows: First, a $\mathrm{C}-\mathrm{V}$ curve is sampled in the target voltage range $V \in\left[V_{1}, V_{2}\right]$ using the given electrode geometry, $e$. A linear regression is then performed to approximate the capacitance as $C=a+b V$ in this voltage range, where $a$ and $b$ are fitting constants. Let $\Delta V=\left|V_{2}-V_{1}\right|$. We then set $M$ to be the product of three factors:

$$
M=p\left(\frac{b \Delta V}{a+b V_{1}}\right)\left(\frac{(b \Delta V)^{2}}{\chi^{2}}\right)
$$

where $\chi^{2}$ is the least squares regression residual, and $p$ is a penalty factor which incorporates design rule constraints and verifies that the device is in the zipping

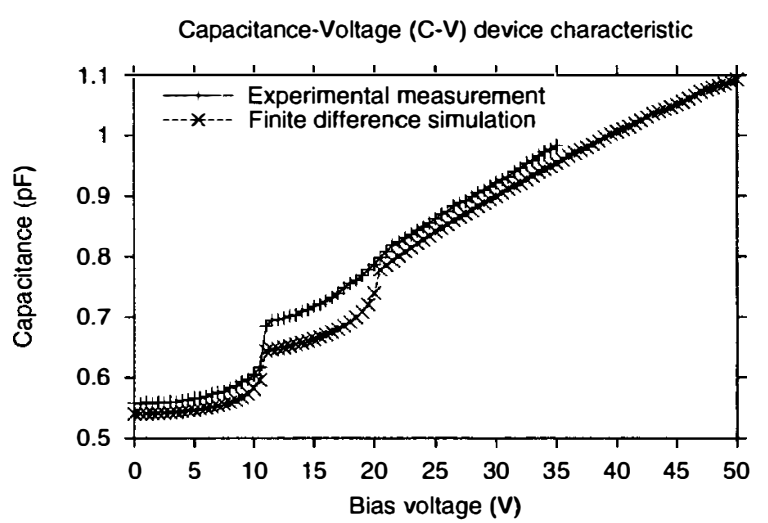

Figure 3. Comparison of measured and simulated $C-V$ characteristic for a MUMPs device with 8 cantilevers in parallel. Jumps in each curve around $10 \mathrm{~V}$ and $20 \mathrm{~V}$ demark transitions between pull-in regimes (Fig. 2). The discrepancy in the data between $10 \mathrm{~V}$ and $20 \mathrm{~V}$ may be due to unmodeled slippage of the cantilever tip. Model and measurement agree well in the linear zipping regime (20-35V) in which the device is designed to operate.

regime. Note that the second factor in Eq. (3) is a measure of the capacitive tuning range, while the third factor is a measure of how closely the $\mathrm{C}-\mathrm{V}$ curve approximates a straight line. Clearly, this is not the only choice, and one can use different functions to emphasize different characteristics.

Since the merit function, $M$, is quite complicated, it is difficult to compute the gradient of $M$. Thus gradientbased optimization routines are not used. Instead a downhill-simplex [14] optimization routine is used to minimize $1 / M$.

\section{RESULTS}

The $\mathrm{C}-\mathrm{V}$ characteristics of prototype MUMPs varactors were measured using an HP4192A. Fig. 3 shows a comparison of measured and simulated $\mathrm{C}-\mathrm{V}$ characteristics for a device with 8 cantilevers in parallel (Fig. 1(d)). The cantilevers are $400 \mu m$ long and $90 \mu m$ wide. The device is optimized to obtain a linear $\mathrm{C}-\mathrm{V}$ characteristic in the zipping regime voltage range, $20-35 \mathrm{~V}$.

The simulation uses film thickness data measured using a WYKO interferometer. Experimental data is only available up to $35 \mathrm{~V}$ due to test setup limitations. Jumps in each curve around $10 \mathrm{~V}$ and $20 \mathrm{~V}$ demark transitions between the regimes depicted in Fig. 2. Initial beam pull-in occurs around $10 \mathrm{~V}$. From $10 \mathrm{~V}$ to $20 \mathrm{~V}$, only the tip of the beam contacts the substrate. Above $20 \mathrm{~V}$, the device is in the zipping regime. The discrepancy in the data between $10 \mathrm{~V}$ and $20 \mathrm{~V}$ may be due to unmodeled slippage of the cantilever tip in the real device. The measured $\mathrm{C}-\mathrm{V}$ characteristic is quite linear in the zipping regime (20-35V) and agrees well with simulation in this voltage range. The offset between the measured and simulated $\mathrm{C}-\mathrm{V}$ characteristic may be due to unmodeled fringing fields and parasitics.

Since experimental data from MUMPs devices verify basic actuator function and modeling accuracy, it is interesting to explore simulated varactor designs with 


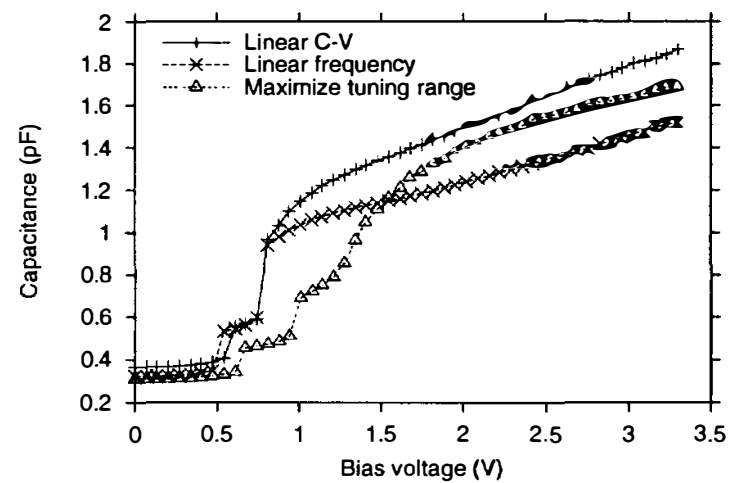

(a)

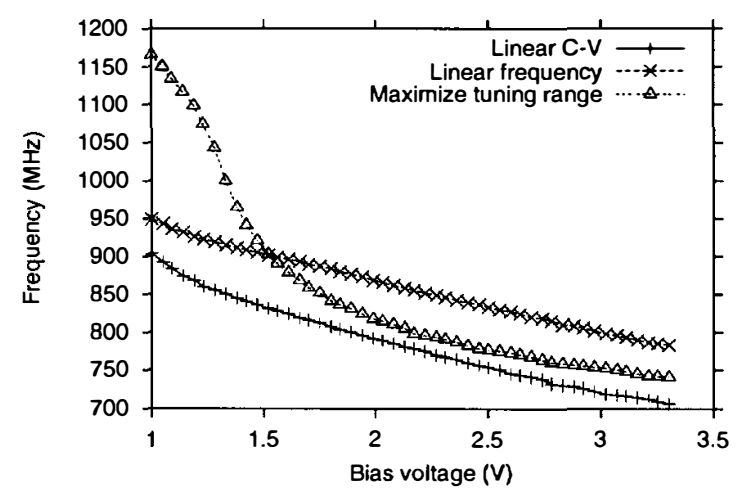

(b)

Figure 4. Simulation of aluminum varactor with 8 parallel cantilevers (0.5 $\mu \mathrm{m}$ thickness, $0.25 \mu \mathrm{m}$ dimple). (a) $\mathrm{C}-\mathrm{V}$ characteristic. (b) Frequency, $\frac{1}{2 \pi \sqrt{L C}}$, with $L=20.4 n H$. The three different curves represent optimizations for linear $C$ - $V$, linear frequency dependence on $V$, and maximal tuning range. The operational voltage range is $1-3.3 \mathrm{~V}$.

high conductivity material and different geometric constraints. There are two geometric parameters to consider, the cantilever thickness and the dimple depth. The cantilever thickness controls the actuation voltage and the dimple depth controls the capacitive tuning range.

Consider an aluminum varactor similar to the baseline MUMPs design except with a $0.5 \mu \mathrm{m}$ beam thickness, $0.25 \mu \mathrm{m}$ dimple depth, and $70 \mu \mathrm{m}$ beam width (width is reduced to prevent electrode contact due to deflection across the width of the beam). We optimize for three different objectives using actuation voltages (1.0-3.3V) consistent with CMOS: (1) linear C-V characteristic, (2) linear $1 / \sqrt{C(V)}$, for linear frequency tuning of an $L C$ tank, and (3) maximum tuning range.

The results are depicted in Fig. 4. The simulations show that it should be possible to actuate varactors with $3.3 \mathrm{~V}$ while optimizing for the three different objectives. Note that an analog tuning range greater than $150 \%$ (2.5:1 ratio) can be achieved if one allows a highly nonlinear $\mathrm{C}-\mathrm{V}$ characteristic.

\section{CONCLUSIONS}

This paper reports results in three areas. First, successful development and fabrication of a stable zipping actuator is reported using flat structures. Second, this actuator is used to implement a tunable capacitance with a programmable $\mathrm{C}-\mathrm{V}$ characteristic determined by the geometry of a shaped electrode. Third, the automated meshed simulation, optimization, and layout procedure used to design the electrode geometry shape is implemented and verified with experimental data.

Prototype devices were fabricated using the MUMPs process from MCNC. A numerical study shows that this approach can be applied to metalized structures, as will be required for integrated RF applications.

\section{ACKNOWLEDGMENTS}

Support for this research was provided by the Defense Advanced Research Project Agency under contract N66001-97-C-8620. The authors wish to thank Darrin Young, Vladimir Rabinovich, and John Gilbert for many useful discussions.

\section{REFERENCES}

[1] D. J. Young and B. E. Boser, "A micromachined variable capacitor for monolithic low-noise VCOS," Proc. Solid State Sensor and Actuator Workshop, Hilton Head, SC, 1996, pp. 86-89.

[2] D. J. Young and B. E. Boser, "A micromachined-based RF low-noise voltage-controlled oscillator," IEEE Custom Integrated Circuits Conference, 1997, pp. 431-434.

[3] L. E. Larson, R. H. Hackett, and R. F. Lohr, "Microactuators for GaAs-based microwave integrated circuits," Transducers '91, pp. 743-746.

[4] J. J. Yao and F. M. Chang, "A surface micromachined miniature switch for telecommunications applications with signal frequencies from DC up to $4 \mathrm{GHz}$," Transducers '95, pp. 384-387.

[5] J. N. Randall, C. Goldsmith, D. Denniston, and T.-H. Lin, "Fabrication of micromechanical switches for routing radio frequency signals," J. Vac. Sci. Technol. B, 146 , 1996, pp. 3692-3696.

[6] K. Sato and M. Shikida, "Electrostatic film actuator with a large vertical displacement," Proc. MEMS 1992, pp. 1 5 .

[7] J. Branebjerg and P. Gravesen, "A new electrostatic actuator providing improved stroke length and force," Proc. $M E M S$ 1992, pp. 6-11.

[8] R. Legtenberg, E. Berenschot, M. Elwenspoek, and J. Fluitman, "Electrostatic curved electrode actuators," Proc. MEMS 1995, pp. 37-42.

[9] J. R. Gilbert and S. D. Senturia, "Two phase actuators: stable zipping devices without fabrication of curved structures," Proc. Solid State Sensor and Actuator Workshop, Hilton Head, SC, 1996, pp. 98-100.

[10] D. A. Koester, R. Mahadevan, and K. W. Markus, MUMPs Introduction and Design Rules, MCNC MEMS Technology Applications Center, 1994.

[11] J. R. Gilbert, G. K. Ananthasuresh, and S. D. Senturia "3D modeling of contact problems and hysteresis in coupled electro-mechanics," Proc. MEMS 1996, pp. 127-132.

[12] G. K. Fedder, S. Iyer, and T. Mukherjee, "Automated optimal synthesis of microresonators," Transducers '97, pp. 1109-1112.

[13] W. Ye, S. Mukherjee, and N. C. MacDonald, "Optimal shape design of an electrostatic comb drive in microelectromechanical systems," J. Microelectromech. Syst., 7 1, March 1998, pp. 16-26.

[14] W. H. Press, et al., Numerical Recipes in C: The Art of Scientific Computing, 2nd edition, Cambridge UP, 1992. 